\title{
AVALIAÇÃO DE CARACTERÍSTICAS DA FIBRA DE GENÓTIPOS DE ALGODOEIRO CULTIVADOS EM SISTEMAS ULTRA-ADENSADO, ADENSADO E CONVENCIONAL
}

\author{
MARTINS, Isaac Silva ${ }^{1}$ \\ SILVA, Isaias Martins ${ }^{2}$ \\ CAMARGO, José Marque ${ }^{3}$ \\ MELO, Lilian Faria ${ }^{4}$ \\ VIEIRA JÚNIOR, Hélvio Carlos ${ }^{5}$ \\ SANTOS, Astúrio Silva ${ }^{6}$
}

RESUMO: O presente experimento foi conduzido com o objetivo de avaliar três genótipos de algodoeiro, cultivados em sistemas ultra-adensado, adensado e convencional, em relação a diferentes características de fibra. $\mathrm{O}$ ensaio foi conduzido de dezembro/2008 a junho/2009, no delineamento em blocos casualizados (DBC) com parcelas subdivididas (SPLIT PLOT), no esquema 3x3 e com 4 repetições, sendo que as parcelas constituíram-se dos espaçamentos (E) $0,90 \mathrm{~m}, 0,76 \mathrm{~m}$ e $0,38 \mathrm{~m}$, e as subparcelas pelos genótipos (G): NUOPAL, DP 90 B e DP OPAL, mantendo uma população de 135.000 plantas por hectare para todos os tratamentos. As parcelas com espaçamento 0,76 m e 0,90 m foram constituídas de quatro linhas, já as parcelas com 0,38 m por seis linhas, todas com $15 \mathrm{~m}$ de comprimento subdivididas em três subparcelas com $5 \mathrm{~m}$ cada. $\mathrm{Na}$ área útil de cada subparcela retirou-se de forma aleatória uma amostra de 20 capulhos, que foram enviadas para o laboratório e analisadas pelo equipamento HVI. Os dados obtidos foram submetidos à análise de variância e as médias comparadas pelo teste de Tukey a 5\% de probabilidade. Pelos resultados observou-se que o espaçamento entre linhas não interferiu nas características de qualidade de fibra dos genótipos analisados.

Palavras-chave: Densidade. Micronaire. Qualidade de fibra. Gossypium hirsutum L.

\section{EVALUATION OF FIBER CHARACTERISTICS OF COTTON GENOTYPES GROWN IN SYSTEMS DENSE AND CONVENTIONAL}

SUMMARY: This experiment was conducted to evaluate the three genotypes cotton grown in ultra-dense, dense and conventional, in relation to different characteristics of fiber. The test was conducted from December/2008 and June/2009 in randomized block design (RBD) split-plot (split plot), in the scheme $3 \times 3$ with 4 replicates, and the plots consisted of spacing (E) $0,90 \mathrm{~m}, 0,76 \mathrm{~m}$ and $0,38 \mathrm{~m}$, the subplots and genotypes (G): NUOPAL, DP $90 \mathrm{~B}$ and DP OPAL, maintaining a population of 135,000 plants per hectare for all treatments. The plots spaced $0.76 \mathrm{~m}$ and $0.90 \mathrm{~m}$ were composed of four lines, since the $0.38 \mathrm{~m}$ plots with six rows, each with $15 \mathrm{~m}$ long divided into three subplots each with $5 \mathrm{~m}$. In the span of each subplot withdrew from a random sample of 20 capsules, which were sent to the laboratory and analyzed by HVI equipment. The data were subjected to analysis of variance and means were compared by Tukey test at 5\% probability. The results showed that the spacing did not influence fiber quality characteristics of the genotypes analyzed.

Keywords: Density. Micronaire. Fiber quality. Gossypium hirsutum L.

\footnotetext{
${ }^{1}$ Mestrando do curso de Pós-Graduação em Ciência do Solo, Faculdade de Ciências Agrárias e Veterinárias - UNESP, Jaboticabal, SP, Departamento de Tecnologia, Via de acesso Prof. Paulo Donato Castellane s/ ${ }^{\circ}$, CEP: 14884-900, Jaboticabal-SP, e-mail: isaac_martins2006@yahoo.com.br

2 Aluno do curso de Graduação em Agronomia, FEIT/UEMG.

${ }^{3}$ Engenheiro Agrônomo, Fundação Educacional de Ituiutaba - FEIT/UEMG - Campus de Ituiutaba, Rua Ver. Geraldo Moisés da Silva, s/nº - Setor universitário, CEP: 38302-192, Ituiutaba-MG.

${ }^{4}$ Mestranda do curso de Pós-Graduação em Ilha Solteira, UNESP.

${ }^{5}$ Professor de Engenharia Agronômica, Fundação Educacional de Ituiutaba - FEIT/UEMG - Campus de Ituiutaba, MG, Rua Ver. Geraldo Moisés da Silva, s/nº - Setor universitário, CEP: 38302-192, Ituiutaba-MG.

${ }^{6}$ Engenheiro Agrônomo, Fundação Educacional de Ituiutaba - FEIT/UEMG - Campus de Ituiutaba, Rua Ver. Geraldo Moisés da Silva, s/nº - Setor universitário, CEP: 38302-192, Ituiutaba-MG.
} 


\section{INTRODUÇÃO}

A cultura do algodão no Cerrado tem evoluído em área e tecnologia, tornando-se o principal fornecedor de fibra do país, com pouco mais de um terço do percentual total, alavancando o desenvolvimento de regiões, fortalecendo a economia das cidades, sendo uma das culturas que mais gera empregos diretos e indiretos.

O algodoeiro é um vegetal da divisão Magnoliophyta, classe Magnoliopsida, ordem Malvales, família Malvaceae, subfamília Malvoideae e gênero Gossypium, segundo o sistema de classificação APG II. Sendo a espécie Gossypium hirsutum L. a mais cultivada no Brasil, sendo conhecido popularmente por algodoeiro comum, algodoeiro americano ou algodoeiro das terras-altas (SOUZA; LORENZI, 2005).

O desenvolvimento do algodoeiro ocorre através de sucessivas fases que correspondem aos seguintes estádios de desenvolvimento: (a) semeadura à emergência (4 a 10 dias); (b) surgimento do primeiro botão floral (30 dias); (c) aparecimento da primeira flor (45 a 60 dias); (d) abertura do primeiro capulho (90 a 120 dias); (e) abertura das maçãs (120 a 180 dias) (BELTRÃO; SOUZA, 2001).

Após a fecundação, a fibra do algodão desenvolve-se na epiderme da semente, sendo que cada fibra é formada por uma célula simples, podendo-se alongar até um milímetro por dia, na fase de máximo crescimento, por meio de deposição de camadas de celulose, até atingir o tamanho final, o qual é função do genótipo e influenciado pelas condições edafoclimáticas. Do florescimento até a abertura dos capulhos pode decorrer um período variável de 50 a 75 dias. O número de fibras por semente varia com a espécie, podendo chegar a 15.000 fibras individuais em uma única semente de Gossypium hirsutum L. (SANTANA et al., 1999).

A fibra é um elemento unicelular, que se desenvolve a partir da semente, inicialmente em forma de pequenos filamentos, que ao passar do tempo vão tomando formato e espessura definitivos (MENDONÇA, 1973). A qualidade da fibra é uma característica que não deve ser ignorada nas análises econômicas sobre o algodão.

A fibra de algodão é classificada com base em suas características intrínsecas e extrínsecas. Entre as intrínsecas, Santana et al. (2000) destacaram: comprimento, uniformidade, maturidade, micronaire (característica que considera tanto a finura quanto a maturidade), resistência, alongamento, reflectância e fiabilidade. Quanto aos aspectos extrínsecos, Penna (2006) citou a cor, a ausência de detritos de folhas e a presença de manchas.

No sistema de produção de algodão no Cerrado predomina a comercialização do produto já beneficiado, ou em pluma, diretamente à indústria têxtil. É usual a instalação de usina de beneficiamento dentro das propriedades, e aqueles produtores que não a possuem contratam esse tipo de serviço. Sendo assim, o preço da pluma é determinado com base nas análises de HVI (High Volume Instrument) e não apenas nas características extrínsecas da fibra (FARIAS et al., 1999). O Brasil passou a utilizar a partir de 2003 os padrões internacionais de classificação, através do Instrumento HVI (SESTREN; LIMA, 2007).

A resistência da fibra é a força, em gramas, requerida para romper um feixe de fibras em tex, que equivale à massa em gramas de 1000 (mil) metros de fibra. Quando expressa em gf tex $^{-1}$ é a resistência ou tenacidade, que é divisão de força máxima pela densidade linear, ou seja, título de fibra (SESTREN; LIMA, 2007).

É de fundamental importância enfatizar que a resistência e o alongamento à ruptura das fibras têm forte relacionamento com a resistência dos fios e tecidos. Fibras fortes geram fios e tecidos resistentes. Consequentemente, promovem menores índices de ruptura nas várias etapas que compõem o processo têxtil, impactando de forma positiva na produtividade, na qualidade e nos custos (SESTREN; LIMA, 2007). 
A resistência e o alongamento à ruptura suportam e absorvem altos impactos e as altas tensões impostas pelas altas velocidades das máquinas que compõem os processos têxteis. Também contribuem quase que diretamente sobre as propriedades físicas dos tecidos, tais como resistência, alongamento, toque, dentre outras (SESTREN; LIMA, 2007).

A mudança de perfil da cotonicultura nacional, passando de um sistema produtivo totalmente manual para o mecanizado, indica uma necessidade dentro da cadeia têxtil, de uma rápida melhoria da qualidade da matéria prima visando atender as necessidades da indústria nacional. Dentro da cadeia têxtil, o setor de fiação e tecelagem é o que tem maior índice de modernização de equipamentos nos últimos anos. Para os novos sistemas de fiação, as características de qualidade da fibra mais importantes são: a resistência, finura (micronaire), comprimento e pureza (BARBOSA; NOGUEIRA JÚNIOR, 2001).

Com o cultivo do algodoeiro com menores espaçamentos, o principal reflexo deverá ser verificado no momento da colheita. O que deve ser respondido é se a qualidade da fibra produzida com este tipo de cultivo atingirá os mesmos níveis de qualidade obtidos com os espaçamentos tradicionais, devido à utilização de um tipo diferenciado de colhedora.

Objetivo desta pesquisa foi avaliar três genótipos de algodoeiro, cultivados em sistemas ultraadensado, adensado e convencional, em relação a diferentes características de fibra: comprimento, resistência e micronaire, para as condições de Ituiutaba-MG.

\section{MATERIAL E MÉTODOS}

O ensaio foi conduzido no período de dezembro de 2008 a junho de 2009 na Fazenda Experimental (FAEXP) da Universidade do Estado de Minas Gerais - UEMG, campus da Fundação Educacional de Ituiutaba-MG - FEIT (FEIT/UEMG), situada no km 762 da BR 365. O solo da área experimental é argiloso classificado como Latossolo distroférrico (EMBRAPA, 1999). Suas coordenadas geográficas são $18^{\circ} 57^{\prime} 03^{\prime \prime}$ de latitude Sul e 49 $31^{\prime} 31^{\prime \prime}$ de longitude Oeste e $560 \mathrm{~m}$ de altitude. Temperatura média anual de $23,9^{\circ} \mathrm{C}$, e umidade relativa do ar em torno de $69,9 \%$ (ABRÃO, 2007).

Os dados climatológicos de precipitação pluvial, registrados no período de dezembro de 2008 a junho 2009, foram obtidos através da Estação Climatológica Principal (ECP) da FEIT/UEMG/ISEPI ligada ao $5^{\circ}$ Distrito Meteorológico, de Belo Horizonte, $\mathrm{BH}$, e pode ser observada na tabela 1.

Tabela 1. Precipitação pluviométrica registrada no período de Dezembro/2008 a Junho/2009.

\begin{tabular}{ccccccc}
\hline & & & & & \\
Dezembro & Janeiro & Fevereiro & Março & Abril & Maio & Junho \\
\hline $358,0 \mathrm{~mm}$ & $351,6 \mathrm{~mm}$ & $202,4 \mathrm{~mm}$ & $215,0 \mathrm{~mm}$ & $71,0 \mathrm{~mm}$ & $280,0 \mathrm{~mm}$ & $31,4 \mathrm{~mm}$ \\
\hline
\end{tabular}

Fonte: ECP da FEIT-UEMG-Ituiutaba-MG

O delineamento experimental adotado foi o de blocos ao acaso com parcelas subdivididas (SPLIT PLOT), no esquema $3 \times 3$ e com 4 repetições. As parcelas constituíram-se dos espaçamentos (E), e as subparcelas dos genótipos (G). Os espaçamentos adotados foram de 0,90 m, 0,76 m e 0,38 m, fixando em 135.000 plantas $\mathrm{ha}^{-1}$ para todos os tratamentos, como mostra na tabela 2. Nas subparcelas, os tratamentos foram constituídos por três cultivares de algodoeiro com arquiteturas e características típicas da agricultura brasileira, foram elas: NUOPAL, DP 90 B e DP OPAL. 
Tabela 2. Tratamentos, Espaçamento, Genótipo, Número de plantas por metro e Densidade de plantas por hectare.

\begin{tabular}{|c|c|c|c|c|}
\hline Tratamentos & Espaçamentos (m) & Genótipo & $\begin{array}{c}\mathrm{N}^{\circ} \text { de plantas (m } \\
\text { linear) }\end{array}$ & $\begin{array}{c}\text { Densidade } \\
\left(\text { plantas ha }^{-1}\right)\end{array}$ \\
\hline 1 & 0,90 & NUOPAL & 12,15 & 135.000 \\
\hline 2 & 0,90 & DP 90B & 12,15 & 135.000 \\
\hline 3 & 0,90 & DP OPAL & 12,15 & 135.000 \\
\hline 4 & 0,76 & NUOPAL & 10,35 & 135.000 \\
\hline 5 & 0,76 & DP 90B & 10,35 & 135.000 \\
\hline 6 & 0,76 & DP OPAL & 10,35 & 135.000 \\
\hline 7 & 0,38 & NUOPAL & 5,35 & 135.000 \\
\hline 8 & 0,38 & DP 90B & 5,35 & 135.000 \\
\hline 9 & 0,38 & DP OPAL & 5,35 & 135.000 \\
\hline
\end{tabular}

As parcelas com espaçamento $0,76 \mathrm{~m}$ e $0,90 \mathrm{~m}$ foram constituídas de quatro linhas de $15 \mathrm{~m}$ de comprimento subdivididas em três subparcelas com $5 \mathrm{~m}$ cada. As parcelas com $0,38 \mathrm{~m}$ foram constituídas por seis linhas de $15 \mathrm{~m}$ de comprimento subdivididas em três subparcelas cada, sendo que cada subparcela possuía um genótipo. Uma linha marginal externa foi semeada em cada lado e em toda a extensão do experimento, para efeito de bordadura.

$\mathrm{Na}$ determinação da área útil de cada subparcela, descartaram-se as duas linhas laterais (uma de cada lado) e um metro em cada extremidade, que ficou assim constituída: três metros centrais, com procedimentos diferenciados em cada subparcela, sendo duas linhas centrais das subparcelas dos tratamentos espaçados de $0,76 \mathrm{~m}$ e $0,90 \mathrm{~m}$ e quatro linhas centrais das subparcelas do tratamento espaçado de $0,38 \mathrm{~m}$.

O preparo do solo foi realizado no dia 09 de dezembro de 2008 e constou de uma aração na profundidade de 0-20 cm, utilizando-se arado reversível, uma gradagem pesada para destruição dos torrões maiores e uma gradagem niveladora. Foram utilizados $2.600 \mathrm{Kg} \mathrm{ha}^{-1}$ de calcário dolomítico para correção da acidez do solo. Os sulcos de semeadura foram abertos com auxílio de enxadas, conforme os espaçamentos adotados $(0,90 \mathrm{~m}, 0,76 \mathrm{~m}$ e $0,38 \mathrm{~m})$, e na profundidade aproximada de $0,05 \mathrm{~m}$. No dia 29 de dezembro de 2008, foi realizada a distribuição das sementes de forma manual, utilizando cerca de 20 sementes por metro linear, garantindo um estande definitivo de 135.000 plantas $\mathrm{ha}^{-1}$, no qual o raleio foi realizado no dia 14 de janeiro de 2009. Para um melhor controle dos patógenos do solo e pragas iniciais, tratou as sementes com carbofuran (Furadan 350 TS) e carboxina e thiram (Vitavax-Thiram 200 SC), de acordo com as recomendações para a cultura.

Foi utilizado na adubação de semeadura $500 \mathrm{~kg} \mathrm{ha}^{-1}$ da formula 4-14-08, com base na análise química do solo ( $5^{\mathrm{a}}$ aproximação para o Estado de Minas Gerais). Em cobertura foram aplicados $250 \mathrm{~kg}$ ha ${ }^{-1}$ de sulfato de amônio após 30 dias da germinação e $133 \mathrm{Kg} \mathrm{ha}^{-1}$ de cloreto de potássio após 45 dias após germinação. Também foi realizada uma aplicação de $0,3 \mathrm{~kg} \mathrm{ha}^{-1}$ sulfato de manganês ( $30 \% \mathrm{Mn}$ ) e de sulfato de zinco $(22 \% \mathrm{Zn})$. Os resultados da análise química do solo, na profundidade de 0 a $20 \mathrm{~cm}$, foram obtidos pelo Laboratório de Solos, do Departamento de Ciências Agrárias da FEIT/UEMG e encontra-se na Tabela 3. 
Tabela 3. Resultados da análise química do solo da área experimental, na profundidade de $0-20 \mathrm{~cm}$. Ituiutaba-MG.

\begin{tabular}{|c|c|c|c|c|c|c|c|c|c|c|c|c|}
\hline $\mathrm{pH}$ & $\mathrm{P}$ & $\mathrm{K}$ & $\mathrm{Al}$ & $\mathrm{H}+\mathrm{Al}$ & $\mathrm{Ca}$ & $\mathrm{Mg}$ & $\mathrm{Sb}$ & $\mathrm{t}$ & $\mathrm{T}$ & M.O & $\mathrm{m}$ & $\mathrm{V}$ \\
\hline $\mathrm{H}_{2} \mathrm{O}$ & \multicolumn{2}{|c|}{$\mathrm{mg} \mathrm{dm}^{-3}$} & \multicolumn{7}{|c|}{$-{ }_{-}$} & $\mathrm{g} \mathrm{kg}^{-1}$ & \multicolumn{2}{|c|}{---\%--- } \\
\hline 5,5 & 14,1 & 141,5 & 0,25 & 7,19 & 3,5 & 0,85 & 4,7 & $\tau$ & 11,9 & 20 & 5 & 40 \\
\hline
\end{tabular}

Fonte: LAB Solos - FEIT-UEMG - 2008

Durante todo o desenvolvimento vegetativo foi aplicado 4 aplicações de ácido bórico (17\% B). O controle de plantas daninhas iniciais foi realizado através de uma capina manual e também foi realizada uma aplicação na forma de jato dirigido no início do fechamento das linhas, utilizando Volcane a base de MSMA (79\%) (na dosagem de 2,0 litros de produto comercial ha ${ }^{-1}$ ), por meio de um pulverizador manual costal de 20 litros de capacidade, equipado com ponta de pulverização tipo leque 110/02.

Foi instalado no mês de novembro de 2008, armadilhas com ferormônios, para monitoramento e controle do bicudo (Anthonomus grandis), uma das principais pragas do algodoeiro. Foi realizado o controle de pragas e doenças (fungos) para manter o bom crescimento e desenvolvimento das plantas. Seguindo recomendações de Righi et al. (1965); Passos (1977) e Gridi-Papp et al. (1992) utilizou-se o regulador de crescimento vegetal, Tuval (cloreto de clormequat) na dosagem de 100 g. i.a ha ${ }^{-1}$, que foi aplicado nas parcelas com pulverizador costal, conforme o monitoramento semanal da altura das plantas, de forma que os espaçamentos correspondessem a dois terços da altura final das plantas.

A colheita foi realizada manualmente no dia dez de junho de 2009 colhendo-se 20 capulhos do terço médio de cada cultivar, nas respectivas repetições. Os capulhos das diferentes cultivares foram submetidos às avaliações de características da fibra com a utilização do aparelho HVI onde foram realizadas as seguintes avaliações: determinação do comprimento de fibra (COM), a resistência da fibra (RESIS), e o índice micronaire (MICR). As análises tecnológicas da fibra provenientes das amostraspadrão foram realizadas pelo laboratório da Minas Cotton - Central de Classificação de Fibra de Algodão de Uberlândia - MG.

Utilizou-se do esquema de análise variância, para a interpretação dos resultados, sendo as médias dos tratamentos comparadas pelo teste de Tukey, a 5\% de probabilidade (BANZATTO; KRONKA, 1995). Os dados foram analisados pelo software "ESTAT", versão 2.0, desenvolvido pelo Pólo Computacional e Departamento de Exatas da UNESP, Campus de Jaboticabal.

\section{RESULTADOS E DISCUSSÃO}

Com base nos resultados apresentados na tabela 4, observa-se que a interação espaçamento $\mathrm{x}$ genótipo foi não significativa $(\mathrm{P}>0,05)$ para resistência, comprimento e micronaire, o que significa que os resultados obtidos com os genótipos independem dos espaçamentos e vice-versa. Quando a interação não é significativa, o efeito de um fator ocorre de forma independente do outro (PIMENTEL-GOMES; GARCIA, 2002). 
Tabela 4. Quadrado da média (Q.M), F da análise de variância (F) e coeficiente de variação da parcela e subparcelas e suas interações (médias de 4 repetições). Ano agrícola 2008/2009. Ituiutaba, MG.

\begin{tabular}{|c|c|c|c|c|c|c|c|}
\hline \multirow[b]{2}{*}{ Causas da variação } & \multicolumn{3}{|c|}{ Resistência } & \multicolumn{2}{|c|}{ Comprimento } & \multicolumn{2}{|c|}{ Micronaire } \\
\hline & G.L. & Q.M. & $\mathrm{F}$ & Q.M. & $\mathrm{F}$ & Q.M. & $\mathrm{F}$ \\
\hline Bloco & 3 & 18,21 & $1,53^{\mathrm{ns}(1)}$ & 1,4051 & $1,38^{\mathrm{ns}(1)}$ & 0,1092 & $1,30^{\mathrm{ns}(1)}$ \\
\hline Espaçamento (E) & 2 & 16,6617 & $2,10^{\mathrm{ns}}$ & 0,2387 & $0,23^{\mathrm{ns}}$ & 0,0399 & $0,71^{\mathrm{ns}}$ \\
\hline Resíduo (A) & 6 & 23,7783 & & 1,0176 & & 0,1684 & \\
\hline Genótipo(G) & 2 & 12,7717 & $1,67^{\mathrm{ns}}$ & 5,2214 & $8,12 *$ & 0,2025 & $5,60 *$ \\
\hline Interação & 4 & 17,4667 & $1,14^{\mathrm{ns}}$ & 0,4561 & $0,75^{\mathrm{ns}}$ & 0,0541 & $0,75^{\mathrm{ns}}$ \\
\hline Resíduo (B) & 18 & 68,9417 & & 0,6427 & & 0,3256 & \\
\hline Total & 35 & & & & & & \\
\hline
\end{tabular}

Verificando a análise de variância, não constatou efeito significativo pelo teste de $\mathrm{F}$ do efeito isolado para o fator espaçamento (tratamento principal) e genótipo (tratamento secundário) a 5\% de probabilidade $(\mathrm{P}>0,05)$ em relação à resistência de fibra (Tabela 4$)$.

De acordo com Jones; Wells (1997) o espaçamento entre fileiras não influencia nas características tecnológicas da fibra, sendo estas características mais influenciadas pela densidade de plantas na linha de semeadura, resultados semelhantes foram observados por Nóbrega et al, (1993); Heitholt (1995); Zandonadi (2008), o que concorda com os dados do presente trabalho onde não se verificou efeito do tratamento espaçamento nem genótipo para a característica resistência de fibra (Tabela 5).

Tabela 5. Resultado das médias de resistência de fibra de algodão em gf tex ${ }^{-1}$ obtidos no ensaio de cultivares em diversos espaçamentos. Ano agrícola 2008/2009. Ituiutaba-MG.

\begin{tabular}{lllll}
\hline \multirow{2}{*}{ Espaçamento $(\mathrm{m})$} & \multicolumn{5}{c}{ Genótipo } & Média \\
\cline { 2 - 5 } & Nuopal & DP 90B & DP Opal & $33,81 \mathrm{a}^{(1)}$ \\
\hline 0,90 & 34,27 & 33,57 & 33,6 & $34,27 \mathrm{a}$ \\
0,76 & 33,52 & 33,75 & 33,05 & $32,65 \mathrm{a}$ \\
0,38 & 32,85 & 31,2 & 33,92 & $33,60 \mathrm{~A}$ \\
\hline Média & $34,30 \mathrm{~A}^{(2)}$ & $32,84 \mathrm{~A}$ & & \\
\hline \multicolumn{5}{c}{ DMS (espaçamento) } \\
DMS (genótipo) & 2,4941 & \\
\hline CV (espaçamento ) (\%) & 2,0395 & \\
CV (genótipo) (\%) & 5,93 \\
(1) Médias seguidas pela mesma letra minúscula, na coluna não diferem, pelo teste de Tukey a 5\%. \\
(2) Médias seguidas pela mesma letra maiúscula na linha não diferem, pelo teste de Tukey a 5\%.
\end{tabular}

As médias para resistência de fibra, que é a força requerida para romper um feixe de fibras de um tex (equivalente à massa em gramas de $1000 \mathrm{~m}$ de fibra), para as fibras colhidas nesse experimento foram classificadas como muito resistentes de acordo com a interpretação proposta por Sestren e Lima, (2007). Em estudo comparativo sobre as características tecnológicas da fibra entre os algodões cearense e norteamericano, Santana et al. (2000) verificou igualdade de condições para a resistência, com média geral de 25,5 $\mathrm{gf} \mathrm{tex}^{-1}$, valor inferior ao encontrado nos genótipos avaliados conforme observado na tabela 5 .

Não foi verificada diferença significativa $(\mathrm{P}>0,05)$ para comprimento de fibra de algodão para os 
diversos espaçamentos, mas houve diferença $(\mathrm{P}<0,05)$ entre os genótipos estudados (Tabela 4), o que discorda dos resultados obtidos por Santana et al. (1999); Zandonadi (2008), que não obtiveram resultados significativos para comprimento de fibras.

Na tabela 6, o genótipo Nuopal $(31,50 \mathrm{~mm})$ apresentou o maior cumprimento de fibra não diferindo do genótipo DP Opal (30,92mm), o menor comprimento foi observado em DP90B (30,19mm) não diferindo de DP Opal (Tabela 6).

Tabela 6. Resultado das médias de comprimento de fibra de algodão (mm) obtidos do ensaio de cultivares em diversos espaçamentos. Ano agrícola 2008/2009. Ituiutaba-MG.

\begin{tabular}{|c|c|c|c|c|}
\hline \multirow[t]{2}{*}{ Espaçamento (m) } & \multicolumn{3}{|c|}{ Genótipo } & \multirow[b]{2}{*}{ Média } \\
\hline & Nuopal & DP 90B & DP Opal & \\
\hline 0,90 & 31,48 & 29,90 & 31,00 & $30,79 \mathrm{a}^{(1)}$ \\
\hline 0,76 & 31,59 & 30,78 & 30,73 & $31,03 \mathrm{a}$ \\
\hline 0,38 & 31,44 & 29,90 & 31,01 & $30,78 \mathrm{a}$ \\
\hline Média & $31,50 \mathrm{~A}^{(2)}$ & $30,19 \mathrm{~B}$ & $30,92 \mathrm{AB}$ & \\
\hline DMS (espaçamento) & & 1,2638 & & \\
\hline DMS (genótipo) & & 0,8354 & & \\
\hline CV (espaçamento) (\%) & & 3,27 & & \\
\hline CV (genótipo) (\%) & & 2,60 & & \\
\hline
\end{tabular}

Os resultados obtidos no presente trabalho discordam de Jost; Cothre (2001) que observaram diminuição no comprimento da fibra nos espaçamentos super adensados $(0,19$ e 0,38 m), já nos espaçamentos convencionais $(0,76$ e $1,01 \mathrm{~m})$ esta redução não ocorreu, o mesmo acontecendo para as demais características de qualidade de fibra estudadas naquele experimento.

Não foi verificada diferença significativa $(\mathrm{P}>0,05)$ para micronaire entre os diversos espaçamentos, mas houve diferença significativa $(\mathrm{P}<0,05)$ entre os genótipos estudados (Tabela 4), sendo que o genótipo Nuopal apresentou o menor valor médio (3,81 $\mu \mathrm{g}$ pol-1), o que significa dizer fibra mais fina, quando comparado com os outros genótipos avaliados (DP Opal e DP 90B) (Tabela 7), o que discorda dos resultados obtidos por Zandonadi (2008), que não obteve resultados significativos para micronaire.

Tabela 7. Resultado das médias de micronaire $\left(\mu \mathrm{g} \mathrm{pol}^{-1}\right)$ obtidos do ensaio de cultivares em diversos espaçamentos. Ano agrícola 2008/2009. Ituiutaba-MG.

\begin{tabular}{lllll}
\hline Espaçamento (m) & \multicolumn{3}{c}{ Genótipo } & \\
\cline { 2 - 5 } & Nuopal & DP 90B & DP Opal & Média \\
\hline 0,90 & 3,81 & 3,92 & 3,96 & $3,89 \mathrm{a}{ }^{(1)}$ \\
0,76 & 3,82 & 3,93 & 3,96 & $3,90 \mathrm{a}$ \\
0,38 & 3,83 & 4,11 & 3,96 & $3,97 \mathrm{a}$ \\
\hline \multicolumn{1}{c}{ Média } & $3,81 \mathrm{~B}^{(2)}$ & $3,99 \mathrm{~A}$ & $3,96 \mathrm{~A}$ & \\
\hline DMS (espaçamento) & 0,2099 & & & \\
DMS (genótipo) & 0,1401 & & \\
\hline CV (espaçamento ) (\%) & \multicolumn{3}{c}{} & \\
CV (genótipo) (\%) & 3,27 & & \\
\hline
\end{tabular}

(1) Médias seguidas pela mesma letra minúscula, na coluna não diferem, pelo teste de Tukey a 5\%.

(2) Médias seguidas pela mesma letra maiúscula na linha não diferem, pelo teste de Tukey a 5\%. 
As fibras colhidas nesse experimento apresentaram valores médios para micronaire entre 3,81 a 3,99, classificando-as como finas, de acordo com a interpretação proposta por Sestren; Lima (2007), ficando ainda, dentro do padrão de preferência do mercado, que exige fibras com índice entre 3,6 e 4,2 (FARIAS et al., 1999).

As pequenas variações em relação às características de fibra de diferentes genótipos de algodoeiro cultivados em diferentes espaçamentos $(0,38 ; 0,76$ e $0,90 \mathrm{~m})$ quando se mantém a mesma população de plantas por unidade de área (135.000 plantas $\mathrm{ha}^{-1}$ ) pode ser em parte justificado pela capacidade de adaptação (interação genótipo-ambiente) das plantas de algodoeiro quando submetidas a diferentes espaçamentos e arranjo de plantas.

\section{CONCLUSÃO}

- Pelos resultados obtidos desse experimento conclui-se que o espaçamento entre linhas não interferiu nas características de qualidade de fibra dos genótipos analisados.

- As fibras mais longas e finas foram obtidas com o genótipo Nuopal, porém não se verificou diferença significativa para resistência entre os genótipos avaliados.

\section{REFERÊNCIAS}

ABRÃO, M.E.G. Determinação dos parâmetros físicos meteorológicos para estimativa da evapotranspiração potencial, caracterização e classificação do clima em Ituiutaba. Belo Horizonte: UEMG - REDEINCA, 2007. 37 p.

BANZATTO, D.A.; KRONKA, S. do N. Experimentação agrícola. $3^{\text {a }}$ ed. Jaboticabal: FUNEP, 1995. $247 \mathrm{p}$.

BARBOSA, M.Z.; NOGUEIRA JÚNIOR, S. Aspectos quali-quantitativos do consumo recente de algodão no Brasil. Informações Econômicas, São Paulo, v. 31, n. 4, p. 37- 39, 2001.

BELTRÃO, N.E.M.; SOUZA, J.G. Fisiologia e ecofisiologia do algodoeiro. In: Algodão: Tecnologia de produção. Embrapa Agropecuária Oeste; Embrapa Algodão. Dourados: Embrapa Agropecuária Oeste. 2001. p. 54-75.

COMISSÃO DE FERTILIDADE DO SOLO DO ESTADO DE MINAS GERAIS. Recomendações para o uso de corretivos e fertilizantes em Minas Gerais, $\mathbf{5}^{\text {a }}$ aproximação. Lavras, 1999. 359p.

EMBRAPA, Centro Nacional de Pesquisa de Solo (Rio de Janeiro, RJ). Sistema Brasileiro de Classificação de Solos. Rio de Janeiro, 1999, 412p.

FARIAS, F.J.C.; et al.. Melhoramento do algodoeiro para o cerrado. In: FUNDAÇÃO MT. Mato Grosso: liderança e competitividade. Rondonópolis: Fundação MT, 1999. p.9-21. (Boletim 3).

GRIDI-PAPP, I.L; et al.. Manual do produtor de algodão. São Paulo: Bolsa de Mercadorias e Futuros, 1992. 158p.

HEITHOLT, J.J. Cotton flowering and boll retention in different planting configurations and leaf shapes. Agronomy journal, v. 87, n.5, p.994-8, 1995.

JONES, M.A.; WELLS, R. Dry matter allocation and fruiting patterns of cotton grown at two divergent plant populations. Crop science. v. 37, p. 797-802, 1997. 
JOST, P.H.; COTHREN, J.T. Phenotypic alterations and crop maturity differences in ultra-narrow row and conventionally spaced cotton. Crop Science, v.41, n.4, p.1150-1159, 2001.

MENDONÇA, E.R. Manual do produtor e do beneficiador do algodão. Brasília: Nacional, 1973. 222 p.

NÓBREGA, L.B. da; et al.. de. Influência do arranjo espacial de plantio e da época da gema apical em algodoeiro herbáceo. Pesquisa Agropecuária Brasileira. v. 28, n. 12, dez.1993. p. 1379-1384.

PASSOS, M.G. Algodão. Capinas: Instituto Campineiro de Ensino Agrícola, 1977. 424 p. PENNA, J.C.V. Melhoramento genético do algodoeiro. In: MORESCO, E. (Org.) Algodão: Pesquisas e resultados para o campo. 2. ed. Cuiabá: CTP, 2006. Cap. 11, p. 262-284.

PIMENTEL-GOMES, F.; GARCIA, C.H. Estatística aplicada a experimentos agronômicos e florestais: exposição com exemplos e orientações para uso de aplicativos. Piracicaba: FEALQ, 2002. 309 p.

RIGHI, N.R.; FERRAZ, C.A.M.; CORRÊA, D.M. VII Cultura. In: INSTITUTO BRASILEIRO DE POTASSA. Cultura e adubação do algodoeiro. São Paulo, 1965. p. 255-317.

SANTANA, J.C.F.; et al.. Características da fibra e do fio do algodão: análise e interpretação dos resultados. In: BELTRÃO, N. E. M. (Coord.). O agronegócio do algodão no Brasil. Brasília: Embrapa Comunicação para Transferência de Tecnologias, 1999. v. 02, p. 857-880.

SANTANA, J.F.C.; WANDERLEY, M.J.R.; BELTRÃO, N.E.M. Tecnologia da fibra e do fio do algodão, análises e interpretações dos resultados. In: EMPRESA BRASILEIRA DE PESQUISA

AGROPECUÁRIA: Centro de Pesquisa Agropecuária do Oeste. Algodão: informações técnicas. Dourados: EMBRAPA-CPAO; Campina Grande-PB: EMBRAPA-CNPA, 2000. p. 232-254. (Circular Técnica, 7).

SESTREN, J.A.; LIMA, J.J. Característica e classificação da fibra de algodão. In: FREIRE, E.C. (Ed.). Algodão no Cerrado do Brasil. Brasília: Associação Brasileira dos Produtores de Algodão, 2007. Cap. 22, p. 765-820.

SOUZA, V.C.; LORENZI, H. Botânica Sistemática: guia ilustrado para identificação das famílias brasileiras, baseado em APG II. Nova Odessa-SP: Instituto Plantarum, 2005.

ZANDONADI, R.F. Desempenho de genótipos de algodão de fibra longa em Campo Verde - MT. Ponta Grossa: Universidade Estadual de Ponta Grossa, 2008. Monografia. 
